\title{
COLOMBIA EN EL POST-ACUERDO Y EL ROL DE LA INFRAESTRUCTURA DE TRANSPORTE
}

\section{Colombia in post-agreement with Farc and the role of transportation infrastructure}

\author{
Carlos Felipe Sánchez Rojas \\ Magister en Economía, Universidad de los Andes. Contacto: cf.sanchez1136@uniandes.edu.co
}

\section{Resumen}

La firma del acuerdo de paz entre el Gobierno Nacional y las Farc traerá grandes desafíos a nivel de infraestructura de transporte, pues los habitantes de regiones que han estado sumidas en el olvido estatal podrán sacar sus productos a los mercados municipales, acceder a productos traídos de otros lugares (reduciendo el auto-consumo), y movilizarse sin las restricciones que habitualmente padecen por la ausencia de carreteras en buen estado. Este artículo analiza los beneficios que traerá la inversión en infraestructura vial a los hogares rurales colombianos.

Palabras clave: Infraestructura de transporte, postacuerdo, mercado, sector rural colombiano.

\section{Abstract}

The peace agreement between the national government and FARC will bring great challenges in terms of transport infrastructure. The inhabitants of regions that have been forgotten by the state, will be able to take their products to municipal markets, access to products imported from other places (reducing self-consumption), and move without the restrictions usually suffer from the absence of roads in good conditions. This article discusses the benefits that will bring investment in road infrastructure for Colombian rural households.

Keywords: Transportation infrastructure, post-agreement, market, colombian rural areas.

\section{Introducción}

Según se espera, antes de finalizar este año se firmarán los acuerdos de paz entre el Gobierno Nacional y las FARC. De las negociaciones no sólo se desprenden temas de justicia, verdad y reparación, sino también una reflexión sobre la responsabilidad del Estado y la sociedad con las regiones en donde habitan la pobreza, el abandono y los grupos armados.

Firmado el acuerdo entrará el país en el llamado 'posconflicto', y con él se evidenciarán realidades sociales deprimentes que en épocas de conflicto se ignoraban. Los planes de desarrollo demandarán más gasto en políticas que generen cohesión social y menos en defensa y seguridad. La mejora de la red vial terciaria, por ejemplo, no sólo traerá mejoras en competitividad, sino también en desarrollo social fomentado por aumentos en la inversión pública y privada, que fortalecen las economías locales y el tejido social en los municipios afectados por el conflicto.

Colombia es un país desigual ${ }^{1}$, y por lo tanto existe una necesidad de cerrar las brechas económicas entre el país formal y el país real. El Estado debe buscar que

1 Según el DANE (2015) la desigualdad de ingresos, entendido como el coeficiente de gini por ingresos se ubicaba en 0,58. 
exista una mejor provisión de oportunidades en todo el territorio, pues las posibilidades que tiene un habitante rural de acceder a educación, salud y demás servicios públicos son escasas.

Las carreteras juegan un papel protagónico a la hora de comunicar centros poblados, por lo tanto, al haber una mejor provisión de ellas aumenta el número de transacciones, se reducen sus costos, incrementa las inversiones públicas y privadas y las oportunidades económicas. Además, al demandar mano de obra, redunda en mayor adquisición de bienes y servicios de parte de los pobladores en los mercados locales, lo que les permite fortalecerse o fomentar la aparición de nuevos.

Sólo con mayor conectividad vial habrá inversiones públicas y privadas que permitan a las familias rurales tener la capacidad de comprar alimentos y acceder a cupos escolares. Y con mayor capacidad de gasto, los hogares pueden darse a la tarea de crear emprendimientos que surtan a los mercados locales de bienes y servicios.

A continuación pretendo mostrar la estrecha relación que tiene el déficit de vías a nivel nacional con la pobreza rural, la carencia de oportunidades y el conflicto armado. Luego, reseño los esfuerzos en política pública que han buscado mejorar la institucionalidad, y finalizo con los retos, que son tan grandes como el rezago que tiene el país en infraestructura de transporte.

\section{Contexto}

Se calcula que el país cuenta con 204.000 km de carreteras, de los cuales cerca de 17.000 km corresponden a vías nacionales (incluidas las vías 4G); 45.000 km son vías de departamentales, y 142.000 km son vías municipales. A pesar que esta categoría representa el 69\% de la extensión vial, hasta el momento no ha existido una iniciativa que modifique la operación de estas vías, como sí sucedió para las vías nacionales cuando se liquidó al Instituto Nacional de Concesiones (INCO) y se designó como responsable de éstas a la Agencia Nacional de Infraestructura (ANI).

Históricamente, Colombia ha presentado una baja inversión en la infraestructura de transporte². Comparando con países del mismo nivel, el nuestro siempre ha estado en las últimas posiciones, como se refleja en el índice de competitividad global, en el área de infraestructura (WEF, 2015). Respecto al pésimo estado de malla vial de las carreteras municipales, este se debe, entre otras razones, a la baja inversión pública y a la confusión de los derechos de propiedad y responsabilidad de administración (el departamento dice que esta le corresponde al municipio y viceversa).

La descentralización institucional en los años noventa implicó la entrega de los derechos de propiedad de la red terciaria a los municipios, una transferencia incompleta, pues de los $142.000 \mathrm{~km}$ de carreteras, 27.500 km aún le pertenecen al INVIAS, 14.000 km a los departamentos y 100.400 km a los municipios. Esta política no estableció ninguna corresponsabilidad a través de programas de financiación o asistencia técnica de gestión a los entes territoriales, imposibilitando una planeación estructurada y de largo plazo. Esto trajo como resultado que solamente $3.615 \mathrm{~km}$ de red terciaria (2.5\%) estén pavimentados, y solo $1.420 \mathrm{~km}(1 \%$ ) en buen estado.

La rehabilitación de la malla vial en la red terciaria es urgente. El estado de la red terciaria imposibilita un arribo de las oportunidades, una presencia permanente de la institucionalidad y los mercados foráneos en el territorio. Sin vías es imposible articular las poblaciones al sistema económico y social. Los hogares del posconflicto demandarán mayor presencia del Estado, y las vías serán instrumentos de política pública necesarios. Por esto vale la pena entender cuál es el papel de las vías en el posconflicto, y cómo ellas contribuirán a transformar la producción, el consumo y los mercados laborales de las comunidades.

\section{Los efectos de las vías en los hogares rurales en el posconflicto}

En el 2011, las mujeres de Barbacoas, Nariño cruzaron las piernas: decidieron entrar en abstinencia sexual para no parir hijos condenados a vivir en el aislamiento y la pobreza hasta que las autoridades públicas les arreglarán la vía que comunica a Barbacoas con Junín, el municipio más cercano (SEMANA, 2015). Los hostigamientos y la presencia de francotiradores de las FARC 
imposibilitaban la intervención vial por parte de los ingenieros militares, relata el artículo de la revista, pues les interesaba mantener el aislamiento y la pobreza para controlar y explotar el territorio, donde la minería ilegal y el narcotráfico eran sus principales fuentes de riqueza (SEMANA, 2015).

A Barbacoas solo se puede llegar de dos formas: la primera es en lancha desde Tumaco, recorrido que toma unas siete horas, o tomando la carretera desde Junín, en un trayecto que puede tardar hasta 20 horas. Así como este municipio nariñense, en Colombia hay muchos otros donde la presencia de grupos armados ilegales impide el acceso de la institucionalidad, perjudicando únicamente a los civiles.

La conectividad y la articulación son retos del posconflicto. Esto solo se logra rehabilitando redes terciarias en los municipios y corregimientos donde el conflicto armado ha sido inminente. Para mostrar la relación que tiene la infraestructura de transporte con el conflicto armado, presento a continuación una estimación cuadrática que explica la relación a nivel municipal entre la densidad vial municipal y la incidencia del conflicto armado. La densidad vial se entiende como la disponibilidad de kilómetros de carretera por kilómetro cuadrado, que en el ejercicio se expresa como la extensión de kilómetros de red terciaria a nivel municipal, por 10 kilómetros cuadrados del área rural.

Por otra parte, se utiliza el Índice de Incidencia del Conflicto Armado (IICA) para medir la presencia de violencia presente en el municipio. Este se entiende como las deviaciones estándar del promedio estandarizado anual, donde entre más desviaciones se aleje del promedio, el municipio se presenta como más violento. Dado que la inversión en infraestructura de transporte ha sido constante durante varios años y el IICA consolida las dinámicas del conflicto durante de 11 años, lo expresado en los gráficos 1 y 2 muestra una relación que ha permanecido a lo largo del tiempo.

Esta relación se evalúa para 1,101 municipios, donde se muestra que a mayor densidad vial, menor es la incidencia del conflicto (ver gráfico 1). Naturalmente, mejores carreteras traen consigo mejor articulación y provisión de bienes y servicios públicos y privados. Las vías son pro-eficiencia y permiten redistribuir de manera más eficiente los recursos a nivel de hogar (Sánchez, 2016). Asimismo, el impacto adicional que trae una vía rehabilitada es menor en regiones ya articuladas que en regiones totalmente aisladas. Es decir, el beneficio que le trae una rehabilitación vial a una familia en Barbacoas, Nariño, no es equivalente al beneficio que le trae a un hogar que reside en Salento, Quindío. Es bastante mayor.

La relación entre densidad vial e IICA muestra que un aumento en densidad vial tiene una relación negativa con la incidencia, pero esta relación pierde magnitud en los municipios más densos (ver gráfico 1). Es decir, municipios con baja incidencia del conflicto tienen mayor variación en los niveles de densidad vial, respecto a los municipios que presentan altos niveles de violencia, donde la mayoría de ellos tienen menores niveles de densidad (ver Gráfico 2). Aunque estas comparaciones solo prueban relaciones y no pretenden explicar causalidad, sí deben generar interés en la realización y ejecución de políticas públicas en el posconflicto.
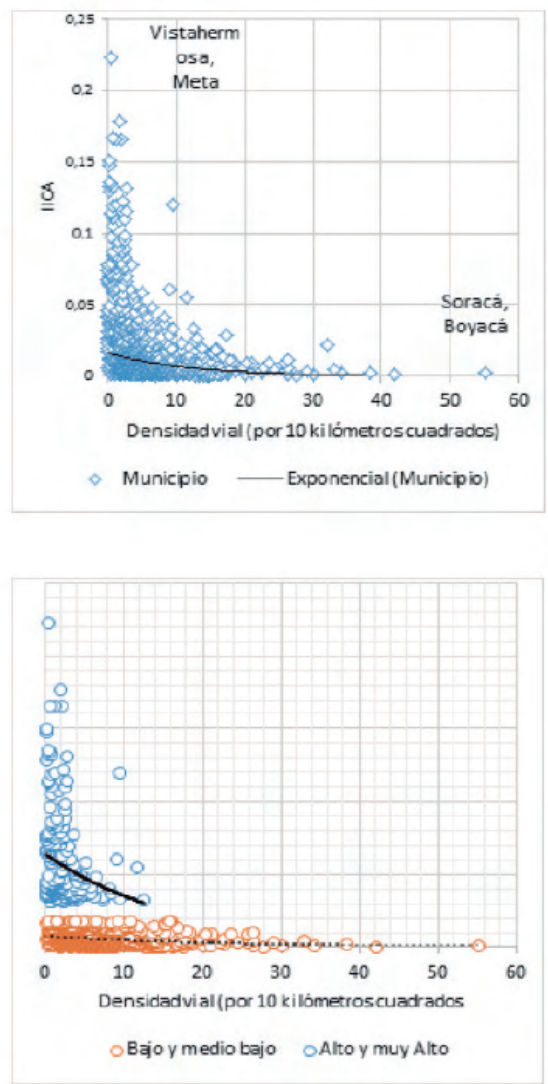

Gráficos 1 y 2: La relación entre conflicto armado y densidad vial 
Dado que el IICA categoriza los municipios por grado de incidencia (bajo, medio bajo, medio alto y muy alto), en el gráfico 2 se puede observar cómo cambia la magnitud de la relación por grupos de incidencia. En los municipios que pertenecen al grupo con mayor incidencia, la relación es mucho más inclinada, a diferencia del grupo con incidencia más baja.

Es decir, rehabilitar los modos de transporte en los municipios donde se presenta mayor incidencia de conflicto armado podrá tener mayores efectos sobre las variables de conflicto que en municipios donde el conflicto no ha estado presente. A manera de ejemplo, una política pública que busque reducir los secuestros y cultivos ilícitos a través de la repavimentación de una vía en Vistahermosa, Meta, tendrá mejores efectos si la misma se efectúa en Soracá, Boyacá.

Entonces las intervenciones viales sí tienen una relación estadísticamente significativa con el conflicto armado, y esta relación es más pronunciada en los municipios con mayor incidencia del conflicto (ver tabla 1). Esta relación se presenta convexa a medida que aumenta la densidad vial. No sólo en Barbacoas, sino de manera generalizada, el conflicto ha impedido inversiones en rehabilitación vial, pues de manera intencionada los grupos al margen de la ley han combatido el acceso del Estado.

Habiendo encontrado una fuerte relación entre la incidencia del conflicto armado y la provisión de la red vial a nivel municipal, y sabiendo que estas intervenciones insertan a las poblaciones aisladas y facilitan la llegada de inversión pública y privada, el siguiente paso será comprender cuáles son los efectos de las vías sobre

\begin{tabular}{|c|c|c|c|}
\hline Variables & $\begin{array}{c}\text { (1) } \\
\text { IICA } \\
\text { Todos los gupos } \\
\end{array}$ & $\begin{array}{c}\text { (2) } \\
\text { IICA } \\
\text { Bejo ym edio bajo }\end{array}$ & $\begin{array}{c}\text { (3) } \\
\text { IICA } \\
\text { Alto ymuyalto }\end{array}$ \\
\hline Densidad vial & $\begin{array}{l}-0.00296^{\text {t12 }} \\
(0.000377)\end{array}$ & $\begin{array}{c}-0.000312^{\text {*te }} \\
(5.80 \mathrm{e}-05)\end{array}$ & $\begin{array}{l}-0.0107^{* 21} \\
(0,00267)\end{array}$ \\
\hline Densidad vial al cuadrado & $\begin{array}{c}6.15 e-05^{\text {tak }} \\
(1.52 e-05)\end{array}$ & $\begin{array}{l}3.77 e-06^{* * t} \\
(1.39 e-06)\end{array}$ & $\begin{array}{r}0.000737^{2+1} \\
(0.000272)\end{array}$ \\
\hline Constante & $\begin{array}{l}0.0315^{* 2 z} \\
(0.00182)\end{array}$ & $\begin{array}{l}0.00952^{2} \\
(0.000327)\end{array}$ & $\begin{array}{l}0.0801^{* * *} \\
(0.00544)\end{array}$ \\
\hline Observaciones & 1,001 & 704 & 167 \\
\hline R-cuadrado & 0.124 & 0.064 & 0.098 \\
\hline
\end{tabular}

Tabla 1: Relación cuadrática entre incidencia de conflicto armado y densidad vial. los hogares rurales. En especial, cómo una mejora vial afecta el ingreso, el consumo y las decisiones de producción, trabajo y uso de tiempo de los individuos.

\section{Efectos de la infraestructura del transporte sobre los hogares rurales colombianos}

En un trabajo realizado por Sánchez (2016) se evalúa para Colombia el impacto que tiene una mejora vial sobre distintas variables de bienestar y competitividad. El autor encuentra que una mejora vial aumenta en promedio 12 kilómetros por hora la velocidad de desplazamiento del hogar rural al casco urbano, y este aumento trae consigo un incremento promedio de 9,6\% y 8,9\% en el ingreso y consumo de los hogares respectivamente.

A su vez, encuentra que las rehabilitaciones viales alteran las fuentes de consumo del hogar rural, pues mejores carreteras permiten sustituir el autoconsumo por consumo comprado, es decir, que el riesgo de no poder comer a causa de choques climáticos disminuye y puede dedicar su tierra a cultivos mercadeables, productivos y rentables. Esta decisión de sustituir cultivos se evidencia cuando existe una caída en el número de productos agrícolas, mostrando una preferencia del hogar rural por especializarse en cultivos productivos, aumentando la competitividad incluso a nivel de regional.

Mayor conectividad vial no sólo genera cambios en las fuentes de consumo, sino también en el uso del tiempo y actividades económicas de los hogares. Estos destinan su tiempo a laborar en actividades agrícolas -incluyendo la ganadería - o no agrícolas, como el comercio, la construcción, entre otras. Del estudio se muestra que la participación del ingreso por actividad económica cambia. Con mejoras viales la participación del ingreso no agrícola sobre el ingreso total incrementa, mientras que la participación del ingreso agrícola disminuye (esto no quiere decir que el nivel del ingreso agrícola caiga, pues los ingresos del hogar incrementan, y los hogares que trabajan productos agrícolas se especializan).

El aumento de la participación no agrícola se debe a que las vías han reducido los costos de transacción, y ello ha permitido la entrada de inversionistas a la economía local, que han aumentado la demanda de mano de obra, por ejemplo, en el sector de construcción, 


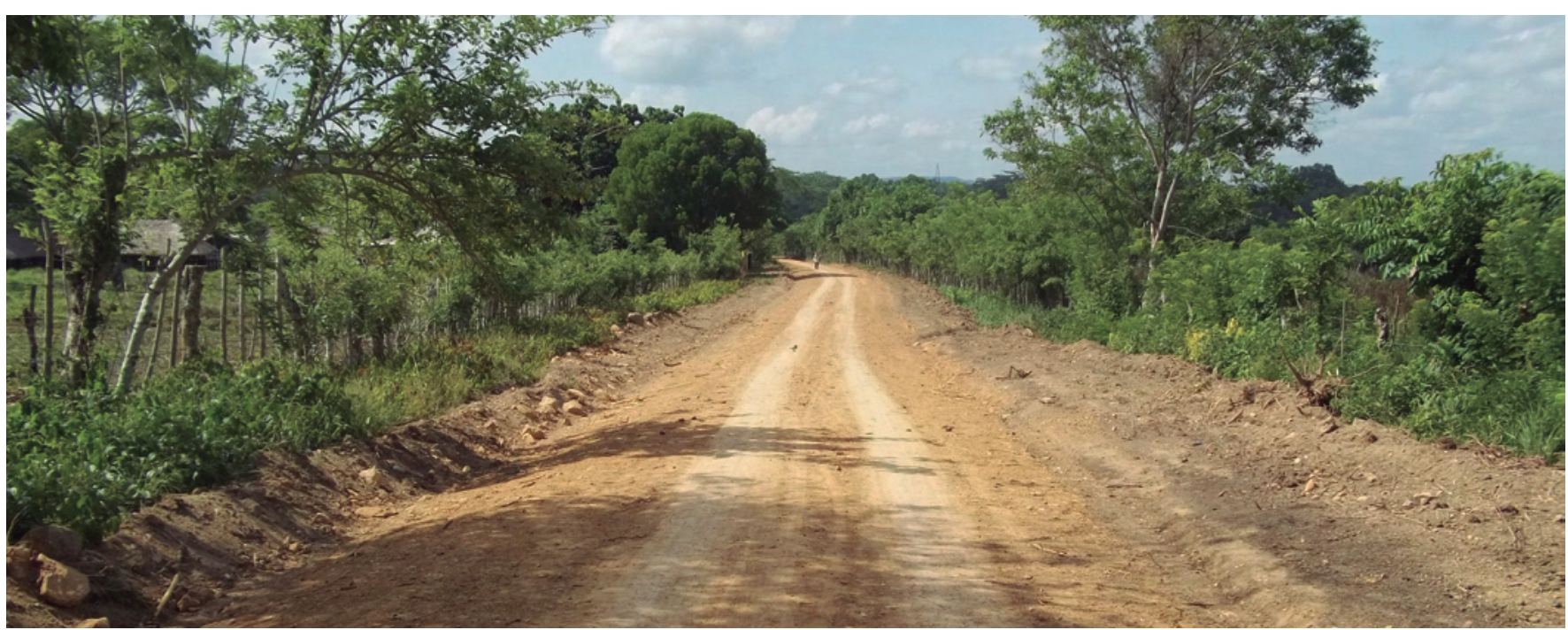

Vía terciaria en Colosó, Sucre. Fuente: Noticias, Sitio oficial del municipio de Colosó, Sucre.

comercio, industria y servicios públicos. Son los mismos hogares que con una rehabilitación en la infraestructura de transporte han convergido en el mercado local, han logrado sacar sus productos agrícolas y aumentado el consumo, generando un aumento en las transacciones económicas y permitiendo el emprendimiento con negocios fuera de la actividad agrícola. Estos procesos se aceleran con la llegada de inversiones extranjeras, pues a ellas también se les da la oportunidad de ejecutar su capital en nuevos territorios, ahora conectados.

Sánchez (2016) utiliza el índice de riqueza que agrega y estandariza los bienes y servicios públicos o privados a que tiene acceso el hogar. Éste índice aumenta 1,37 desviaciones estándar del promedio. Un efecto relevante pues muestra que no solo los hogares están consumiendo su nuevo ingreso, sino que, además, en la comunidad se están haciendo inversiones en servicios públicos.

Por último, se divide la muestra entre hogares rurales que tuvieron una intervención por primera vez y hogares que ya habían tenido intervención vial. Al comparar los resultados, los hogares intervenidos por primera vez muestran mayores impactos en comparación al segundo grupo. Lo anterior indica que realizar rehabilitaciones viales en las comunidades donde hay menor acceso y mayor incidencia del conflicto armado, las hace socialmente más eficientes que con inversiones en rehabilitación en comunidades ya articuladas a economías foráneas.
Entonces, si se quiere acercar las comunidades del posconflicto a los servicios el Estado y los mercados, la mejor política que se puede ejecutar es la rehabilitación de vías de red terciaria. Estas políticas públicas orientadas a la infraestructura de transporte lograrán dinamizar la actividad económica rural, volviéndola más competitiva y permitiendo a la población acceder a mejores servicios de educación y salud, como sucedió en Bangladesh (Levy, H. et al., 1996).

\section{Esfuerzos de política pública}

Desde los inicios de la presidencia de Juan Manuel Santos, se ha empezado a transformar la institucionalidad en el tema de infraestructura de transporte. Se purgó la forma de contratar con el Estado, pues se eliminaron prácticas anticompetitivas que también incentivaban los sobrecostos de las obras. Se liquidó el Instituto Nacional de Concesiones (INCO) para crear la Agencia Nacional de Infraestructura (ANI); se establecieron nuevas modalidades de contratación, como las Alianzas Público Privadas (APPs), y se estructuró el Plan Maestro de Transporte Intermodal (PMTI), plan que trasciende los cuatrienios presidenciales y busca rehabilitar vías férreas, carreteras y acuapistas para los próximos 20 años.

Respecto a la institucionalidad de red terciaria, luego de la Ley 103 de 1993 y la liquidación del Fondo Nacional de Caminos Vecinales (FNCV), se transfirió a los 
entes territoriales la responsabilidad de administrar y ejecutar los proyectos de inversión. Esta transferencia fue incompleta, pues no estuvo soportada con planes de acompañamiento en gestión y financiación. Semejante desarticulación ha permitido que las dinámicas de competencia desleal y corrupción se repitan.

Ahora, el Departamento Nacional de Planeación (DNP), en representación del gobierno nacional, ha establecido el CONPES 3857 de red terciaria, que establece los primeros lineamientos para cambiar estas malas prácticas.

\section{Seis componentes estructuran el documento:}

1) Levantamiento de un inventario vial de red terciaria georreferenciado, que se actualice constantemente. El levantamiento se sugiere participativo, donde las comunidades se comprometen a cumplir a cabalidad sus tareas.

2) Una metodología de priorización para poder ordenar proyectos viales, según la conveniencia social que estos le traigan a la nación.

3) Un manual de diseños y materiales regionalizado para determinar los requerimientos técnicos de una vía y, por tanto, su costo diferenciado.

4) Establecer modelos de cofinanciación que incentiven a los entes territoriales a invertir recursos en rehabilitación vial, de la mano del gobierno.

5) Estructurar los contratos-tipo para estandarizar los costos de contratación y reducir el riesgo de sobrecostos.

6) Una evaluación a las prácticas propuestas en el documento.

EI CONPES es un primer paso para reorientar e impulsar las intervenciones viales de red terciaria de manera organizada, estructurada y eficaz. Es un esfuerzo por purgar las malas prácticas adquiridas luego de la liquidación del FNCV y un intento por reorganizar y fortalecer la forma en que se gestionan estos proyectos, vitales para el posconflicto. No obstante, aunque el documento es un gran avance, la naturaleza de red terciaria, al ser extensa y diversa, necesita el apoyo de las comunidades y los territorios. Por ende, es necesario una institucionalidad que involucre a las comunidades, tal como sucedió en el Perú, y así como lo relata la experiencia de levantar un inventario vial en Antioquia.

\section{Retos}

El mayor reto, que no se abarca en el CONPES, es generar una estructura institucional robusta que soporte el largo plazo. Vale la pena preguntarse cómo será el proceso de actualización del inventario. ¿Se realizará una gran actualización cada cuatro años? O acaso de manera descentralizada puede permitirse una alternativa más práctica. También, es necesario tener en cuenta que el modelo de priorización de proyectos viales, cuando las vías son de propiedad de los territorios no debe ser un modelo generalizado pues continuaría los problemas de gestión y administración que hoy existen entre el gobierno central y los territorios. Estos desafíos deben evaluarse antes de convertirse en problemas para la gestión pública.

El país necesita una institucionalidad en red terciaria que centralice la información del inventario y que tenga la capacidad de responder a las distintas exigencias técnicas, como también requerimientos ambientales, legales y financieros de los proyectos de intervención en los 142.000 kilómetros de carreteras. Por esto, no sólo los municipios sino las comunidades locales a nivel veredal deben jugar un papel protagónico en el proceso. Así lo entendió Perú y su institucionalidad (PROVIAS descentralizado) ha operado de forma ejemplar.

Las comunidades no sólo son fuentes de empleo a la hora de intervenir sino ellas también pueden emprender un proyecto de intervención vial a nivel local que les permite organizar y crear pequeñas empresas que redunden en el fortalecimiento del desarrollo local. Ellas también funcionan como alertas tempranas lo que permite minimizar el costo de una intervención, a su vez permiten tener la información actualizada primera mano no sólo del estado de su malla vial, sino también de su entorno. Al ser las responsables y directamente beneficiadas de usufructuar este bien público, al sistema se le facilita una mayor eficiencia y eficacia. 
Al contar con una extensa red proveída a lo largo de la geografía, se necesita entonces una institucionalidad descentralizada que promueva un mayor protagonismo de las comunidades. Una institución que contribuya y asista a las regiones al avalar proyectos que cumplan los requerimientos técnicos, legales, financieros y ambientales que los proyectos demanden. Además, que ésta misma centralice la información vial de red terciaria que envían los territorios. Contar con una institucionalidad de éstas características fortalece y complementa la participación de las comunidades, asimismo logra centralizar y llevar un recuento del estado vial actualizado con cada encargo de intervención que presentarían las comunidades. Asimismo, una institución participativa permitirá a las comunidades del posconflicto acercarse y ser partícipes de la institucionalidad.

\section{Conclusión}

El rol de la infraestructura de transporte es el rol dar oportunidades. Las consecuencias del conflicto armado ha recaído en el área rural y ha sumado a generaciones de familias en la pobreza y la victimización. A ellas no solamente les ha tocado vivir entre la escasez y el conflicto, sino que se han visto en la obligación de tomar decisiones inverosímiles como dejar de parir para acabar con los círculos de la pobreza.

El reclutamiento forzado, la mortalidad infantil, la deserción escolar, incluso el embarazo adolescente y en general la pobreza son causados parcialmente por la ausencia de inversión en servicios públicos y privados que en regiones aisladas no llegan por los altísimos costos de transporte, sumado al riesgo latente del conflicto armado. Del posacuerdo llega el posconflicto y con él, sin los costos del conflicto, una oportunidad para el Estado de invertir en territorios abandonados. La intervención de red terciaria es primordial, pues invertir en carreteras significa permitir inversiones en salud, educación, seguridad, asistencia técnica y apoyar a que los hogares reasignen más eficientemente sus recursos pues las rehabilitaciones viales aliviaron sus limitaciones y ampliaron sus oportunidades.

La política pública se ha esforzado por recuperar la institucionalidad en la infraestructura de transporte. En los años recientes, la inversión en red terciaria ha aumentado de sobremanera, al igual que la creación del CONPES permite dar los lineamientos para organizar la red terciaria. No obstante, la naturaleza de la red demanda una institucionalidad diligente y abierta, que se acomode a los territorios, en especial a las comunidades si se quiere una sostenibilidad a largo plazo. Así lo realizó Perú y ha logrado funcionar. Sostener 142.000 $\mathrm{Km}$ de red terciaria demanda el protagonismo de las comunidades.

El Estado llega donde los costos le permiten. El posconflicto, sin duda, reduce los costos de acceso a las comunidades pero le obliga también a generar gruesas

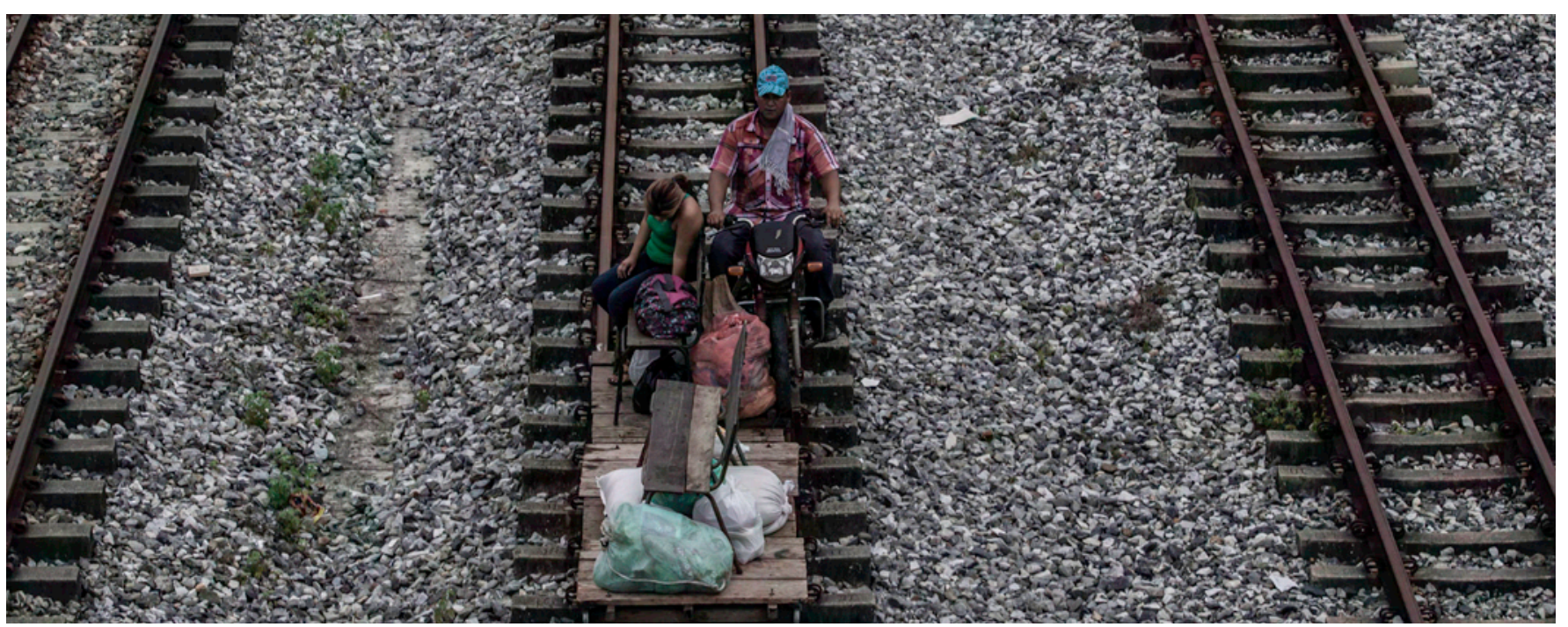

Vías del tren, Barrancabermeja, Santander. Fotografía: Alejandro Gómez, Comunicaciones y Marca-ELCA, Uniandes. 
inversiones no solo para reconstruir tejido social, sino también para proveer servicios públicos con los que hoy las poblaciones aisladas no cuentan. Sin duda, contar con la participación del sector privado será necesario, y para que ello ocurra, la reducción en los costos de transporte, es decir, la rehabilitación de red terciaria, es primordial. Inversiones viales traen externalidades positivas que fortalecerán la asistencia del Estado, la presencia de los mercados y las oportunidades de los hogares en el posconflicto.

\section{Referencias}

DANE (2015). Pobreza monetaria y multidimensional en colombia. Boletín técnico (2014).

DNP (2016). Índice de incidencia del conflicto armado. Grupo de proyectos especiales. Bogotá.

Fedesarollo (2013). Infraestructura del transporte en Colombia. Cuadernos Fedesarrollo 45.

Levy, H., \& Voyadzis, C. (1996). Morocco Impact Evaluation Report: Socioeconomic Influence of Rural Roads.Fouth Highway Projects. Impact Evaluation report.

Sánchez, C.F. (2016). El impacto de la infraestructura vial en los hogares rurales colombianos. ¿Hacia dónde van las vías?. Documento CEDE. Bogotá

Revista Semana (2015). Vías para los olvidados. Publicaciones Semana.

Villar, L., \& Ramirez, J. M. (2014). Infraestructura regional y pobreza rural. FEDESARROLLO working paper.

World Bank (2009). Reshaping Economic Geography. Washington: World Bank.

World Economic Forum. Reporte del índice de competi tividad global. WEF Report. 